\title{
Frequency response characterization of ironless inductive position sensors with long cables
}

\author{
Adrian Grima ${ }^{1,2}$, Mario Di Castro ${ }^{1}$, Alessandro Masi $^{1}$ and Nicholas Sammut ${ }^{2}$ \\ ${ }^{1}$ EN-SMM-MRO Group, European Organization for Nuclear Research (CERN), Geneva, Switzerland \\ ${ }^{2}$ Department of Micro and Nanoelectronics, Faculty of ICT, University of Malta, Msida
}

\begin{abstract}
The two linear position sensors used to determine the position of the European Organization for Nuclear Research; Large Hadron Collider collimator's jaws with respect to the beam are the linear variable differential transformer and the ironless inductive position sensor. The latter was designed as an alternative to the former since the linear variable differential transformer exhibits a position error in magnetic environments. The ironless inductive position sensor is an air cored, high-precision linear position sensor, which is by design immune to external DC or slowly varying magnetic fields. Since the ironless inductive position sensor is required to have no on-board electronics, the raw signal has to be carried through long cable lengths and this may lead to performance degradation. This paper focuses on a set of experimental measurements conducted to assess the ironless inductive position sensor's sensitivity at different frequencies with long cable lengths. This is critical for the sensor`s correct operation in the Large Hadron Collider`s collimators. Furthermore, to gain a better understanding, the ironless inductive position sensor's frequency response is compared with a commercial off-the-shelf linear variable differential transformer.
\end{abstract}

\section{Introduction}

Some of the many requirements of designing and operating a position sensor in the harsh environment of the Large Hadron Collider (LHC) [1] are; long lifetime and robustness, radiation hardness and magnetic field immunity. These include areas subjected to electromagnetic interference, radiation, high temperatures or mechanical stress. These requirements are rather common for several critical applications such as particle accelerators, nuclear plants and plasma control [2]-[5]. For example, tiny fractions of the energy of the stored beam in the Large Hadron Collider is sufficient to quench a super-conducting LHC magnet or to lead to the destruction of acceleration components. The LHC Collimators are part of the complex, machine protection system of the LHC at the European organization for nuclear research (CERN). Hence, it is very important that the collimation position measurements are not influenced by nuclear radiation or by magnetic fields [6] coming from surrounding devices [7]-[9].

Collimators are designed to physically narrow the beam of particles in the transverse plane. This results in cleaning the excess particles in the outer part of the beam halo. An LHC collimator consists of two 1-meter long jaws of graphite, copper or tungsten and can be moved perpendicular to the beam to reduce its transverse size. Each collimator has several electrical devices installed, particularly: stepper motors for the jaw positioning; linear position sensors for the jaws' position monitoring; and temperature sensors for the thermal monitoring and cooling. Given that the jaws carry out the primary function of the collimator, the most important requirement for it to be operational is the position accuracy. The target uncertainty of the position reading is one tenth of the nominal beam size at the collimator $(200 \mu \mathrm{m})$, thus, the jaws have to be measured with a $20 \mu \mathrm{m}$, maximum target position uncertainty.

Due to the high level of radiation expected in the proximity of the collimators (several MGrays/year), no electronics can be embedded in the sensors or in the motors. In fact, all the electronics are placed hundreds of meters away and are connected to the sensor via a cable. This provides a challenge to the sensor and electronics design because the cable and its properties affect the signals sent or received. Furthermore, each collimator is equipped with multiple linear position sensors. In order to avoid interference from nearby magnetic fields operating at the same frequency the linear position sensors are operated at different frequencies.

The Ironless Inductive Position Sensor (I2PS) is a linear position sensor used in such environments [10]-[12]. In this case, the I2PS is used to determine the jaw position of the collimators [13], [14] of the LHC. It is noted that this sensor is radiation-hard, immune to magnetic fields and offers good uncertainty and long lifetime. The I2PS does not need to have embedded electronics and it can be operated at different frequencies with either a current or a voltage supply.

This work aims at expanding the frequency characterisation and investigate the effect of the long cable on the I2PS performance. The I2PS will be 
compared to a Commercial Off-The-Shelf (COTS) Linear variable differential transformer, which is another linear position sensor that is used in the collimators.

\section{The ironless inductive position sensor design}

The I2PS, as can be noted from Figure 1, is made of five coaxial coils; two supply coils, two sense coils and one moving coil. As the name implies the moving coil is the winding connected to the movable link whose position needs to be measured. This coil, as can be noted from the diagram, is short circuited such that there will be an induced current in this coil. The two supply coils are fed a sinusoidal current or voltage signal and generate two equal-but-opposite magnetic fluxes. Comparing the structure to an electric bridge, when the moving coil is at the centre, the electric bridge is at its equilibrium condition. This is because the net induced current in the moving coil is zero (since there are two equal-andopposite magnetic fluxes the sum of the induced currents adds up to zero). This means that the mutual inductances between the moving coil and the supply coils are equal and hence the voltages on the two sense coils are also equal. As the moving coil is displaced from the centre, the equilibrium condition is broken and the two sense voltages are different.

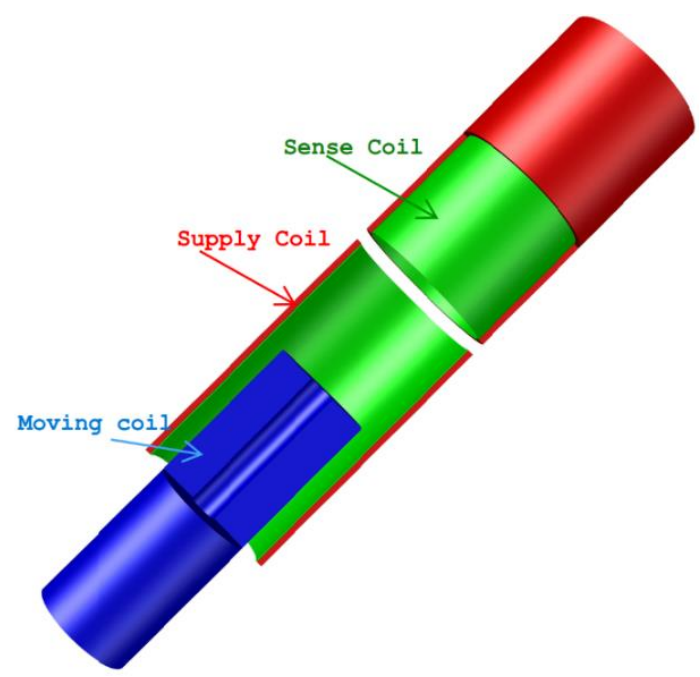

Fig 1. A diagram depicting the structure of the ironless inductive position sensor structure.

The position of the moving coil can be extracted by a differential reading of the fundamental harmonic of the sense coils' voltage. This signal is multiplied by a windowing function prior to being processed by the sinefit algorithm. The amplitude of the signal is then adjusted taking into account the gain correction factor of the window. Finally, the three-parameter sine-fit algorithm is used to demodulate the position from the sensor's voltage [15], [16]. The position reading of the I2PS is then obtained by a ratiometric decoding of the two secondary voltages.
The relationship between the input current and the output voltage is very well described in [10]. The sense coils' voltages for the I2PS are:

$$
\begin{aligned}
& V_{3}=j \omega\left(M_{31}-M_{32}\right) I+j \omega M_{35} I_{5} \\
& V_{4}=j \omega\left(M_{41}-M_{42}\right) I+j \omega M_{45} I_{5}
\end{aligned}
$$

where:

- $V_{3}$ is the voltage output from one of the sense coils of the I2PS,

- $\quad M_{i j}$ are the mutual inductances between $i^{t h}$ and $j^{\text {th }}$ coils,

- $\quad I$ is the input current (since the I2PS is using a current supply) and

- $\quad I_{5}$ is the current induced in the moving coil.

Hence, the transfer function can be derived as:

Where

$$
\frac{V_{3}(s)}{I(s)}=\frac{A}{\left(\mathrm{~L}_{\mathrm{c}}\right) \mathrm{s}+\mathrm{R}_{\mathrm{mc}}}
$$

$$
\begin{gathered}
A=\left(\left(\mathrm{M}_{31}-\mathrm{M}_{32}\right) \mathrm{R}_{\mathrm{mc}}\right) \mathrm{s}+\omega^{2}\left(\mathrm{M}_{35}\left(\mathrm{M}_{51}-\mathrm{M}_{52}\right)\right. \\
\left.-\mathrm{L}_{\mathrm{c}}\left(\mathrm{M}_{31}-\mathrm{M}_{32}\right)\right)
\end{gathered}
$$

To derive the transfer function with the cable the process is divided into two parts: the supply coils and the sense coils. Considering the supply coil side first, the circuit resolves to the one presented in Fig 4.

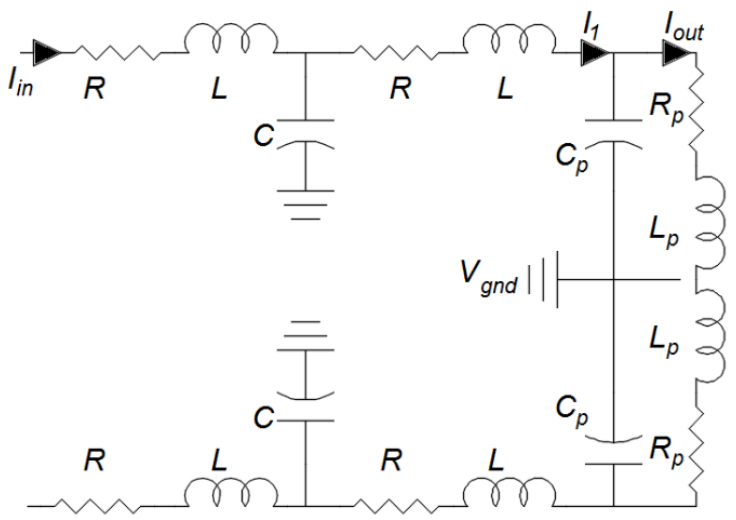

Fig 2: Cable model with I2PS supply coils as load

Hence, it can be derived that:

$$
\begin{aligned}
& \frac{I_{\text {out }}}{I_{\text {in }}}=\frac{I_{\text {out }}}{I_{1}} \frac{I_{1}}{I_{\text {in }}} \\
& =\frac{1}{s^{4}(a)+s^{3}(b)+s^{2}(d)+s(e)+1}
\end{aligned}
$$

Where:

$$
\begin{aligned}
a & =C_{p} L_{p} L C \\
b & =C_{p} R_{p} L C+C_{p} L_{p} R C \\
d & =L C+C_{p} R_{p} R C+L_{p} C+C_{p} L_{p} \\
e & =R C+R_{p} C+C_{p} R_{p}
\end{aligned}
$$

and $C_{p}, L_{p}$ and $R_{p}$ are the capacitance, inductance and resistance respectively of one supply coil. $L$ is the inductance of the cable, similarly $R$ and $C$ are the resistance and capacitance of the cable as a function of the cable`s length. 
Figure 3 shows the equivalent circuit for the output side. In this case, the sense coil's parasitic capacitances need to be taken into consideration as the source impedance, while the acquisition input impedance needs to be taken as a load. In this case, the load is resistive but there are instances where bias capacitors are also added and hence need to be equated in the load.

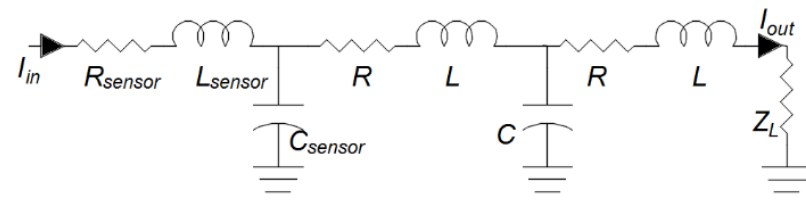

Figure 3: Cable model with I2PS sense coil as supply and acquisition bias resistor as load

The transfer function resolves to:

$$
\begin{aligned}
& \frac{I_{\text {out }}}{I_{\text {in }}} \\
& =\frac{1}{(\mathrm{LC}) \mathrm{s}^{2}+(\mathrm{RC}+\mathrm{ZC}) \mathrm{s}+1} \\
& \quad \times \frac{1}{(\mathrm{LC}) \mathrm{s}^{2}+\left(\mathrm{RC}_{\text {sensor }}+\mathrm{Z}_{\mathrm{L}} \mathrm{C}_{\text {sensor }}\right) \mathrm{s}+1} \\
& \frac{1}{Z_{\text {in }}}=\frac{F}{\mathrm{G}+2 \mathrm{R}+\mathrm{Z}+\mathrm{R}_{\text {sensor }}(F)+\mathrm{sL}_{\text {sensor }}(F)}
\end{aligned}
$$

and

where:

$$
Z_{L}=\frac{s L+R+Z}{s\left(C_{\text {sensor }} L\right)+\left(Z C_{\text {sensor }}+C_{\text {sensor }} R\right) s+1}
$$

$$
\begin{aligned}
B= & 2(R C L)+L C Z \\
D= & R C Z+R^{2} C+2 L \\
E= & (C Z+C R)+(2 R+Z) C_{\text {sensor }} \\
F= & \left(L^{2} C_{\text {sensor }} C\right) s^{4}+\mathrm{B} s^{3} \\
& \quad \quad \quad\left(\mathrm{D} C_{\text {sensor }}+\mathrm{CL}\right) s^{2}+(E) s+1 \\
G= & L^{2} C s^{3}+\mathrm{Bs}^{2}+\mathrm{D} s
\end{aligned}
$$

Finally multiplying Equations 4 and 5 with $\mathrm{Z}$ gives $\frac{V_{\text {out }}}{V_{\text {in }}}$. From the theoretical model, it can be deduced why it is advantageous to operate the I2PS with a current supply. Supplying the sensor with a voltage supply implies that the amplitude of the supply coil voltage will be a function of the cable length. This is not the case when the I2PS is operated with a current supply.

\section{Frequency Experiments and Results}

The theoretical model is tested with a sinusoidal signal and the output voltages of $\pm 9.4 \mathrm{~V}$ and $\pm 4.69 \mathrm{~V}$ with a 50 $\mathrm{mA}$ and $25 \mathrm{~mA}$ peak current supply respectively. This voltage is obtained at a frequency of $1 \mathrm{kHz}$ with the mutual inductances set for the moving coil to be at position $0 \mathrm{~mm}$. The electromagnetic model presented in [10] can be used to generate the mutual inductances at other positions. When a $1 \mathrm{~km}$ return cable is added to the model there is a $4 \mathrm{~V}$ attenuation as can be noted in Table 1 .
Table 1: Table comparing the sense coil voltages obtained from the experimental test-bench with different cable lengths with respect to those obtained from the model and from the simulation. All the values are obtained with a current supply of $50 \mathrm{~mA}$ at $1 \mathrm{kHz}$.

\begin{tabular}{|c|c|c|c|}
\hline & \multicolumn{2}{|c|}{ Sense coils Voltage } & \\
\hline Cable Length (m) & Exp (V) & Model (V) & Diff (\%) \\
\hline $\mathbf{0}$ & $\mathbf{8 . 5}$ & 9.4 & 10 \\
\hline $\mathbf{2 0 0}$ & $\mathbf{8 . 3}$ & 9.4 & 9 \\
\hline $\mathbf{4 0 0}$ & $\mathbf{8 . 1}$ & 8.4 & 4 \\
\hline $\mathbf{6 0 0}$ & $\mathbf{7 . 5}$ & 7.3 & 3 \\
\hline $\mathbf{8 0 0}$ & $\mathbf{6 . 7}$ & 6.3 & 6 \\
\hline $\mathbf{1 0 0 0}$ & $\mathbf{5 . 9}$ & 5.4 & 9 \\
\hline
\end{tabular}

There is an average difference of $0.43 \mathrm{~V}$ between the experimental values and the theoretical ones, i.e. $7 \%$ difference. This difference is acceptable and may be attributed to experimental error and minor physical phenomena that are ignored in the analytical model. The same readings were repeated with different cable lengths, supply current and frequency giving approximately the same discrepancies.

For an in-depth frequency analysis of the sensor with cables an empirical approach is taken so as to avoid ignoring un-modelled parasitic effects. A test bench was set up where a network analyser is used with an active probe. This offers probing with negligible circuit loading due to its low input capacitance. Furthermore, since frequency response of the I2PS with a long cable is being investigated and since the long cable used in the collimator environment has 24 pairs, the different cable lengths were achieved by bridging the different pairs in a $200 \mathrm{~m}$ cable. It was made sure that each $200 \mathrm{~m}$ portion is disconnected when not used (since it was noted that this affects the results).

The frequency response of the I2PS with different cable lengths shows that with increasing cable length, the frequency range of operation of the sensor and the gain decrease. As is shown in Fig 4, without cable the I2PS exhibits a response similar to that of a high pass filter, with a $+20 \mathrm{~dB} / \mathrm{dec}$ gradient at low frequencies and a cutoff at $1.3 \mathrm{kHz}$. Some attenuation starts at $100 \mathrm{kHz}$.

The average gain is $1.5 \mathrm{~dB}$. When the cable is attached, the response becomes similar to that of a band pass filter. Figure 2 also shows that an increase in cable length leads to a narrower bandwidth and a lower gain. It is important to note that there is a $5.5 \mathrm{~dB}$ difference in gain without cable compared to when the sensor is connected to a $1 \mathrm{~km}$ cable as is shown in Fig 4. This $5.5 \mathrm{~dB}$ difference in gain translates to $275 \mu \mathrm{m}$ of position change. It is also important to note that the cable does not have a very drastic impact on the gain at low frequencies up to $900 \mathrm{~Hz}$.

The same procedure is repeated for the LVDT. Although the LVDT is the basis for the I2PS design, its method of operation and construction is different. This can also be noted from their frequency response. The LVDT has a much lower gain and a smoother flat-band, 
unlike the I2PS, which is characterised by its low frequency gradient. Furthermore, a change in the cable does not change the bandwidth of the sensor. Increasing the cable length attenuates the gain at the lower frequencies.

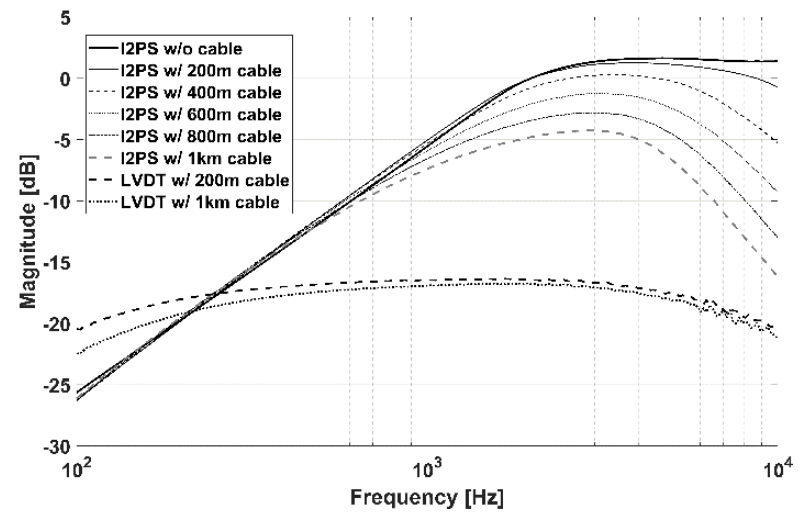

Fig 4. Comparison between the effect of the cable on the frequency response of the I2PS and the LVDT, as obtained from test bench

Fig 5 and Fig 6 show the frequency response when the moving coil is moved at $10 \mathrm{~mm}$ intervals between \pm 30 $\mathrm{mm}$. The moving coil position is varied to investigate the effect of the cable on the position range. This shows that even though the cable length reduces the bandwidth, the voltage variation due to the moving coil is not perturbed. It is further noted that the operating range for the I2PS is between $500 \mathrm{~Hz}$ and $2.5 \mathrm{kHz}$, as is shown in Fig 5 and in Fig 6. Additionally, it can also be seen that a change in the frequency also changes the amplitude of the sense coils. This change depends also on the cable's length since the flat top is achieved much faster with the $1 \mathrm{~km}$ cable as opposed to the $200 \mathrm{~m}$ one. Translating these graphs to position change, a change between a $200 \mathrm{~m}$ cable to a $1 \mathrm{~km}$ one at $2 \mathrm{kHz}$ translates to $725 \mu \mathrm{m}$ and a change of frequency from $1 \mathrm{kHz}$ to $2 \mathrm{kHz}$ translates to $400 \mu \mathrm{m}$ of change.

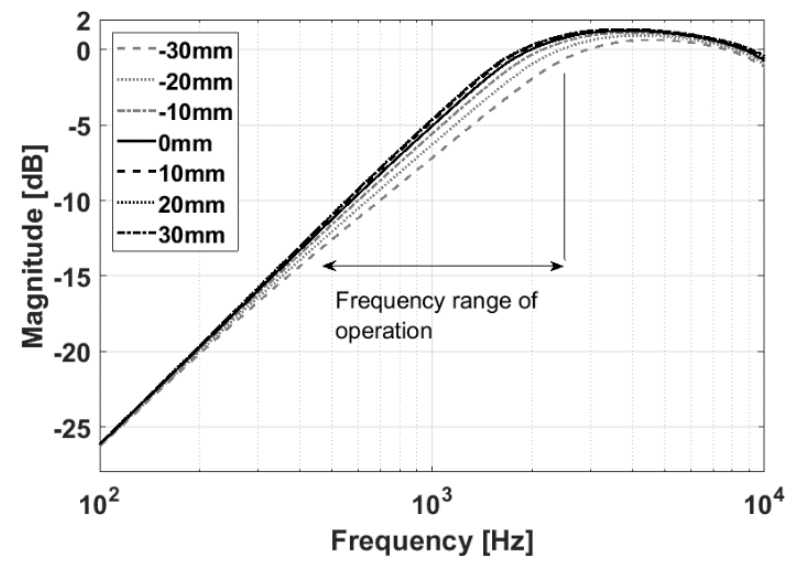

Fig 5. Varying the moving coil position with a $200 \mathrm{~m}$ cable

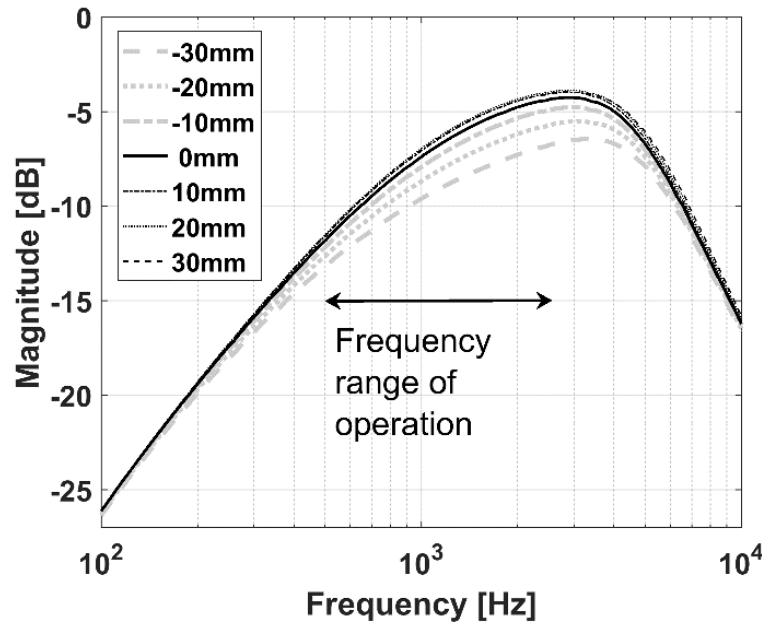

Fig 6. Varying the moving coil position with a $1 \mathrm{~km}$ cable.

As can be noted from Fig 4, this change could be avoided if the frequency of operation selected was on the flat band of the frequency response. This is not possible since at those frequencies the sensor is not sensitive to change as can be noted from Fig 5 Fig 6. It is also important to note that if a new sensor is designed which is sensitive in the pass band of the frequency response of Fig 4 ; it must be ensured that the cable does not attenuate this frequency since in this case; the cable's effect is noted mostly at high frequencies. It can therefore be deduced that the best option is to have a sensor whose passband starts from low frequencies.

The experimental frequency response of the sensor attached to the supply circuitry, cable and acquisition were also compared with the simulation results. Fig 7 compares the simulation with the experimental measurements for three different cable lengths with the moving coil set in the centre.

This discrepancy is due to the factors that have not been taken into account in the simulation. Namely, the windings' imperfections due to the multilayers, the mechanical imperfections of the shield, and the lack of homogeneity of the electrical conductivity in the shield and the imperfections of the sealant used to seal the sensor, which influence significantly the parasitic components of the sensor at high frequency. It has been stated in [17] that the sensor has a strong sensitivity with respect to the winding's imperfection, especially on the moving coil. Since the simulation is strongly dependent on the geometry, it can lead to big discrepancies.

The simulated frequency response is similar in shape to that obtained from the experimental test bench. It also behaves in a similar way, i.e. when the cable length is increased; a decrease in gain and bandwidth is noted. The main difference is the lower cut-off frequency, which is higher for the simulations. Overall, the simulation and the test bench measurements yield similar behaviours. Hence, it can be concluded that within this parametric space, simulations can be used when the sensor's electronics are changed. 


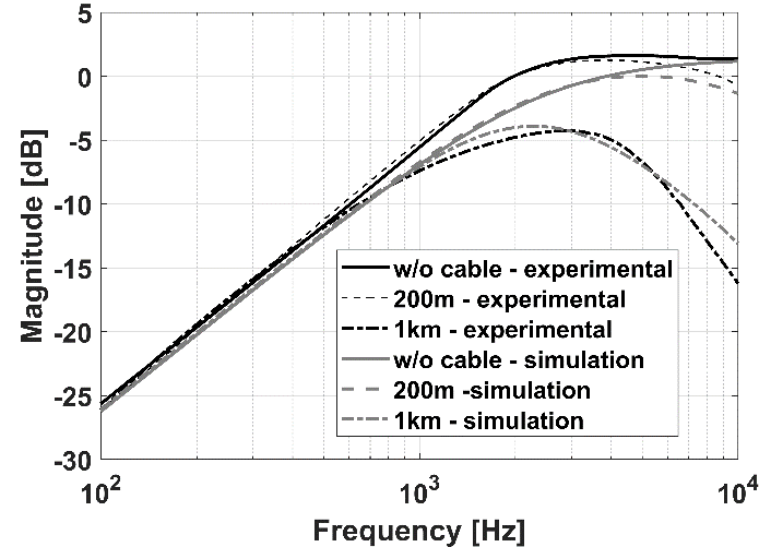

Fig 7. Effect of cable on the frequency response with different cable lengths with comparison to simulation

The focus henceforth is to redesign the sensor or the electronics to flatten the response and increase the sensitivity range. This allows the I2PS to be operated in a wider range of frequencies as well as eliminate the frequency response changes when the cable is attached. The challenge for the current application is that the I2PS installed are operated at different frequencies and each collimator is located at a different distance from the electronics. Each collimator has 7 I2PS installed each operating at a different frequency. As detailed in [1], [14] and [18] the I2PS are operated at $250 \mathrm{~Hz}$ intervals. Hence, the frequency range is just enough to cater for the current collimators. New, special collimators with 14 linear position sensors are designed where the I2PS should be used due to high electromagnetic interference but the frequency range is not enough to cater for 14 I2PS.

\section{Conclusion}

The results show that the cable parameters do affect the position reading significantly. Increasing the cable lowers the sense coils' voltage and reduces the bandwidth. Additionally, a frequency range for operation is defined. The results from the I2PS are compared to a commercial off-the-shelf LVDT, which has a flatter frequency response and is less sensitive to changes in cable parameters.

Moreover, this study shows that greater care needs to be taken in the design and optimisation stage of the I2PS in order to obtain a sensor with a wider or flatter frequency response in its range of operation. Finally, it confirms the need for accurate calibration of the sensor with cables when changes are made.

\section{References}

1. O. Brüning, Large Hadron Collider Design Report. Geneva : CERN: European Organization for Nuclear Research, 20041. The LHC Main Ring.

2. L. Evans and P. Bryant, "LHC machine," Journal of Instrumentation, vol. 3, (08), pp. S08001, 2008.

3. N. Mokhov et al, "Tevatron beam halo collimation system: design, operational experience and new methods1," Journal of Instrumentation, vol. 6, (08), pp. T08005, 2011.

4. W. Scandale et al, "The UA9 experimental layout," Journal of Instrumentation, vol. 6, (10), pp. T10002, 2011.

5. G. Anelli et al, "The totem experiment at the cern large hadron collider," Journal of Instrumentation, vol. 3, (08), pp. S08007, 2008.

6. A. Danisi, "Simulation of DC interfering magnetic field effects on the LHC collimators' LVDT positioning sensors," M.Sc., Electronic Engineering Department, University of Naples" Federico II", Naples, Italy, 2009.

7. A. Masi et al, "Study of magnetic interference on an LVDT: FEM modelling and experimental measurements," Journal of Sensors, vol. 2011, 2011.

8. M. Martino et al, "Design of a linear variable differential transformer with high rejection to external interfering magnetic field," IEEE Trans. Magn., vol. 46, (2), pp. 674-677, 2010.

9. A. Masi and R. Losito, "LHC collimators low level control system," IEEE Trans. Nucl. Sci., vol. 55, (1), pp. 333-340, 2008.

10. A. Danisi, "Ironless inductive position sensor for harsh magnetic environments," 2013.

11. A. Danisi et al, "Design optimization of an ironless inductive position sensor for the LHC collimators," Journal of Instrumentation, vol. 8, (09), pp. P09005, 2013.

12. A. Grima et al, "Influence of External Conductive Objects on the Performance of an Ironless Inductive Position Sensor," IEEE Sensors Journal, vol. 17, (14), pp. 4500-4507, 2017.

13. R. Assmann et al, "LHC collimation: Design and results from prototyping and beam tests," in Particle Accelerator Conference, 2005. PAC 2005. Proceedings of The, 2005, pp. 1078-1080.

14. T. Weiler et al, "LHC collimation system hardware commissioning," in Particle Accelerator Conference, 2007. PAC. IEEE, 2007, pp. 1625-1627.

15. S. Wu and J. Hong, "Five-point amplitude estimation of sinusoidal signals: With application to LVDT signal conditioning," IEEE Transactions on Instrumentation and Measurement, vol. 59, (3), pp. 623-630, 2010.

16. M. Martino, R. Losito and A. Masi, "Analytical metrological characterization of the three-parameter sine fit algorithm," ISA Trans., vol. 51, (2), pp. 262$270,2012$.

17. A. Danisi, "Ironless inductive position sensor for harsh magnetic environments," 2013.

18. A. Danisi, "Ironless Inductive Position Sensor for Harsh Magnetic Environments.", École polytechnique fédérale de Lausanne, 2013. 